\title{
Printing on liquid elastomers
}

\author{
Lingzhi Cai, ${ }^{a}$ Joel Marthelot, ${ }^{\text {ab }}$ Claudio Falcón, ${ }^{c d}$ Pedro M. Reis ${ }^{e}$ and \\ P.-T. Brun ${ }^{a}$ \\ ${ }^{a}$ Department of Chemical and Biological Engineering, Princeton University, \\ Princeton, New Jersey 08540, USA. E-mail: lingzhic@princeton.edu, \\ pbrun@princeton.edu \\ ${ }^{b}$ Aix-Marseille University, CNRS, IUSTI \\ 13013 Marseille, France \\ ${ }^{c}$ Departamento de Física, Facultad de Ciencias Físicas y Matemáticas, \\ Universidad de Chile, Casilla 487-3, Santiago, Chile \\ ${ }^{d}$ Millennium Nucleus on Smart Soft Mechanical Metamaterials, Santiago, Chile \\ ${ }^{e}$ Flexible Structures Laboratory, École Polytechnique Fédérale de Lausanne, \\ 1015 Lausanne, Switzerland
}

In recent years the research community has paid significant attention to geometrically engineered materials. These materials derive their unique properties from their structure rather than their chemistry alone. Despite their success in the laboratory, the assembly of such soft functional materials remains an outstanding challenge. Here, we propose a robust fluid-mediated route for the rapid fabrication of soft elastomers architected with liquid inclusions. Our approach consists of depositing water drops at the surface of an immiscible liquid elastomer bath. As the elastomer cures, the drops are encapsulated in the polymer and impart shape and function to the newly formed elastic matrix. Using the framework of fluid mechanics, we show how this type of composite material can be tailored.

Natural soft materials are often architected at all scales and shaped in periodic structures achieving properties that cannot be matched by manmade constructs. ${ }^{1-4}$ Adapting these concepts to our technological needs has prompted research across many disciplines, from photonic and phononic materials to mechanical metamaterials. In such materials, inclusions, e.g. liquid drops, bubbles, particles and voids, play a central role as their careful positioning enables the modification of the bulk material properties to tailor the optical, ${ }^{5-8}$ acoustic, ${ }^{9,10}$ surface $^{11}$ and mechanical $^{12-16}$ properties of the composite.

Despite the development of various fast-prototyping and microfluidic techniques ${ }^{17-22}$ for the production of compliant materials ranging from artificial tissues to flexible sensors, ${ }^{23-25}$ it becomes increasingly clear that advances in soft functional materials are hindered by the lack of methodology to efficiently fabricate architected soft materials. In particular, recent studies have demonstrated that embedding droplets in the bulk of an elastic matrix can modify its mechanical properties, ${ }^{26,27}$ increase its thermal conductivity ${ }^{28,29}$ or enable its actuation. ${ }^{30}$ Yet, these approaches typically rely on shear to disperse the liquid phase into the elastomer, resulting into emulsions with polydisperse distributions of disorganized drops. Additionally, the possibility of encapsulating droplets selectively in defined areas of the elastomer is virtually unexplored. Overall, there is a lack of strategies to easily tailor the location, size and shape of droplets embedded in composite elastomers. Controlling those features would open the path to new and improved functionalities.

Here, we propose a simple droplet-based methodology to functionalize soft elastomers. We control the deposition of small water droplets at the surface of an elastomeric liquid bath using the framework of fluid mechanics. Upon crosslinking, the interface of the elastomer keeps the imprint of the droplets as it presents millimetric cavities whose position and geometry can be tailored. We study the shapes and spatial arrangements of both the drops and cavities that are produced at the interface. In particular, we identify a regime in which the water drops are encapsulated in the elastic matrix and sit right below a thin membrane at the end of the process. Our work offers an efficient route to fabricate structures where liquid inclusions are used. This fast fabrication technique could be impactful for practical applications such as compartmentalized reactors, ${ }^{31}$ drug delivery, scaffolded biocompatible materials, ${ }^{23,32}$ and encapsulated active matter. ${ }^{33}$

Fig. 1a outlines our approach: droplets of deionized water are deposited at the surface of a liquid elastomeric polymer (VPS Elite Double Vinylpolysiloxane). VPS (Elite Double 32, Elite Double 22, Elite Double 16, Elite Double 8, Zhermack) is prepared by mixing with a weight ratio of $1: 1$ (base to curing agent) using a 


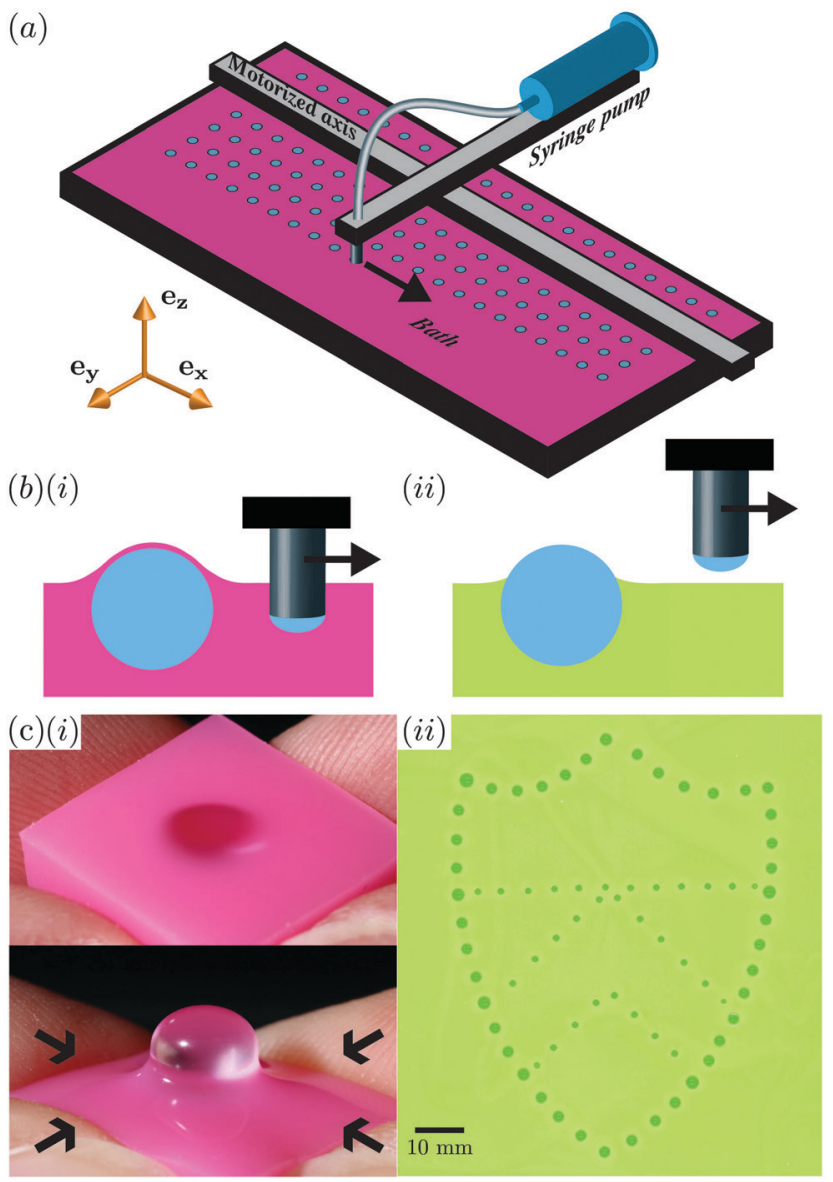

Fig. 1 (a) Water drops are deposited at the surface of a liquid elastomeric bath while the polymeric solution cures. Injection is performed through a needle that travels across the bath. (b) Injection below (resp. above) the bath interface leaves water droplets encapsulated (resp. at the airelastomer interface). (c) Photographs of the elastic materials formed after the elastomer has cured: (i) an encapsulated droplet bulges when compressing the elastic matrix. (ii) The interface of an elastomer patterned with cavities of different sizes forming the Princeton University logo.

centrifugal mixer (ARE-310, Thinky) for $10 \mathrm{~s}$ at $2000 \mathrm{rpm}$ (clockwise), and then $10 \mathrm{~s}$ at $2200 \mathrm{rpm}$ (counterclockwise). Water is injected at a given flow rate in the range $0.17<Q\left[\mathrm{~mL} \mathrm{~min}^{-1}\right]<$ 4.00 through a needle with inner radius $r$ connected to a syringe pump (New Era NE-1000). The needle is mounted on a translational stage (AxiDraw V3.0) moving at a constant speed $u(4.0<$ $\left.u\left[\mathrm{~mm} \mathrm{~s}^{-1}\right]<90.0\right)$ in the $x-y$ plane of the bath. Water is less dense than VPS so that the droplets float in the bath. Yet, depending on the depth of injection, the dispersed phase may sit (i) below a thin elastic membrane or (ii) at the free surface of the bath, as shown in the sketches in Fig. 1b. Curing is performed at room temperature $\left(20^{\circ} \mathrm{C}\right)$. As the polymer cures, the elastomer sets in a shape matching the contour of the drops. After curing the polymer can easily be peeled from its container and the resulting elastic material can be manipulated. In particular, these composite materials are highly deformable as shown in Fig. 1c(i). This route of assembly can easily be tailored to create specific target patterns using drops of different sizes and spacing (see Fig. 1c(ii)).
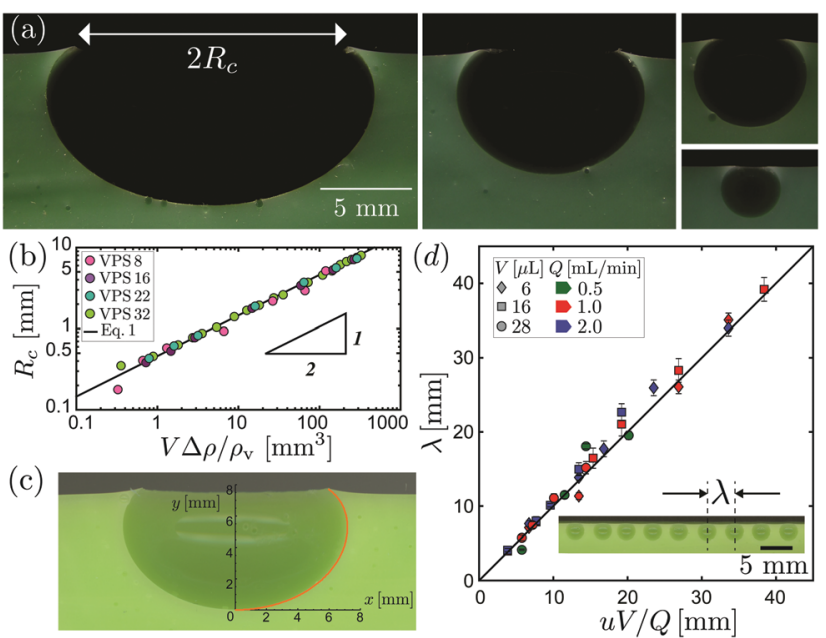

Fig. 2 (a) Shape of cavities obtained with water drops of volume $V=1800$, $400,100,20 \mu \mathrm{L}$ (from left to right). Images are obtained under a microscope. Polymer samples are cut in halves with a precision knife. (b) Apparent radius of the cavities $R_{\mathrm{c}}$ plotted versus the volume of the droplets $V$ scaled by the ratio of the density difference between VPS and water, $\Delta \rho=\rho_{\vee}-\rho_{0}$ and $\rho_{0}$. (c) Comparison between the numerical prediction obtained solving the Laplace equation using shooting method and the actual shape of the cavity. (d) Distance between droplets $\lambda$ plotted versus the needle speed $u$ normalized by the dripping frequency $Q / V$. (Inset) A cross-section of the polymer shows the uniform spacing of cavities.

In Fig. 2, we describe the mechanisms that set the shape and position of the droplets in VPS. The cavities formed by small droplets are quasi-spherical while larger volumes lead to the formation of puddles (Fig. 2a). Particular attention is given to $R_{\mathrm{c}}$, the apparent radius of the cavity defined in Fig. 2a. In Fig. $2 \mathrm{~b}$ we report the values of $R_{\mathrm{c}}$ for water drops with density $\rho_{0}$ deposited in four different polymeric baths with density $\rho_{\mathrm{v}}=\rho_{0}-\Delta \rho$. The density of the liquid polymers was measured after mixing the base with curing agent: (Elite Double 32) 1.22, (Elite Double 22) 1.19, (Elite Double 16) 1.17, (Elite Double 8) $1.07 \mathrm{~g} \mathrm{~cm}^{-3}$. In our experiments we varied the volume of the drops in the range $20<V[\mu \mathrm{L}]<1800$. As evident from Fig. 2b, we find that the apparent radius scales as $R_{\mathrm{c}} \sim V^{1 / 2}$. This geometrical feature is determined by the combined effects of capillary forces and buoyancy. ${ }^{34,35}$ In our experiments, we observe that the upper portion of the drops sitting above the air-elastomer interface is mostly flat. ${ }^{36}$ We approximate this volume as a circular disc with radius $R_{\mathrm{c}}$ and thickness $\ell$. Therefore the volume of water above the bath surface is $V_{\text {disc }}=\pi R_{\mathrm{c}}{ }^{2} \ell$. In turn, balancing weight and buoyancy in our system yields $\rho_{0} V=\rho_{\mathrm{v}}\left(V-V_{\text {disc }}\right)$, such that:

$$
R_{\mathrm{c}}=\sqrt{\frac{\Delta \rho}{\rho_{\mathrm{v}}} \frac{V}{\pi \ell}}
$$

In Fig. $2 b$ we compare eqn (1) to the experimental data, finding excellent agreement with $\ell=1.5 \mathrm{~mm}$. We can relate this value of $\ell$ to the capillary length of water in air. Additionally, we found that the shape of the cavities can be predicted upon integration of Laplace equation. ${ }^{37}$ The value of $R_{\mathrm{c}}$ inferred from eqn (1) is 
used to set the boundary conditions of the problem, and the numerical solution is chosen so as to match the submerged volume of the droplet $\rho_{0} V / \rho_{\mathrm{v}}$. The obtained solutions align well with experiments as depicted in Fig. 2c, where the prediction is superposed onto the photograph. The consistency between the predicted and actual droplet shape hinges on a time scale separation. The time for the liquid system to achieve mechanical equilibrium is indeed much shorter than that of curing. ${ }^{44}$ Hence, the droplet shape is at mechanical equilibrium when the polymer sets (with minimal change in volume).

Having rationalized the shape of the cavities, we turn to describe their volume and the distance $\lambda$ that separates them. We first focus on the case where drops are printed from a needle positioned above the free surface. In this case, the mechanism setting the drop size is as follows. As water is dispensed, a drop forms at the tip of the needle and increases in volume. Dripping occurs when the weight of the drop exceeds the capillary force holding the drop at the needle tip. ${ }^{38}$ The corresponding critical volume is $V=2 \pi \alpha \ell^{2} r$, where $\alpha$ is a known factor that depends on the ratio $r / \ell .^{38,39}$ Therefore, the volume of the droplets $V$, and subsequently the cavity radius $R_{\mathrm{c}}$, can be changed by varying the inner radius of the needle $r$.

For a given flow rate $Q$, the dripping frequency is $f=Q / V$. In turn, the distance between droplets is the distance traveled by the nozzle between two consecutive dripping events $\lambda=u / f=u V / Q$. In Fig. $2 \mathrm{~d}$ we report the values of the wavelength of the pattern, $\lambda$, that progressively forms at the surface of the elastomer matrix. The wavelength $\lambda$ obtained for different values of $u, r$ and $Q$ collapse onto a line with slope 1, confirming that $\lambda=u V / Q$.

When printing above the free surface, the droplet generation is dominated by capillary effects so that the size of the drops and thus that of the resulting cavities is millimetric (given that the capillary length is $\ell \simeq 1.5 \mathrm{~mm}$ ). In particular, the radius of the cavities is typically larger than that of the needle. To obtain smaller droplets, we inject water at the air-elastomer interface while the needle translates across the bath. This strategy aims at detaching the drops from the needle using the bath as an anchor point. In Fig. 3a we show typical photographs of a time sequence of this printing regime, obtained with a high-speed camera (Movie S1 in ESI $\dagger$ ). The height of the needle tip relative to the air-elastomer interface is denoted by $h$. In Fig. $3 b$, we report the wavelength $\lambda$ of the printed pattern as a function of the printing depth $h$ for rectilinear printing trajectories. When the needle tip is far above the interface, drops drip from the needle owing to gravity. As a result the height of the nozzle $h$ does not affect $\lambda$ (regime $\mathrm{I}$, discussed earlier in the paper). When the needle tip is brought closer to the interface, the hydrodynamics involved in the drop formation is more complex (regime II). Water droplets come into contact with the viscous polymeric bath before detaching from the needle (Fig. 3a). The droplets are then sheared as the needle moves relative to the bath, until the breakup occurs. This process leads to the deposition of fixed volumes of water at regular intervals. In this regime II, we observe that the wavelength $\lambda$ of the breakup pattern is sensitive to the position of the needle tip and decreases linearly with $h$. When the tip of the needle comes into contact with the polymeric bath, $h=0$, a minimum in the wavelength is observed (as marked by a (*) in Fig. $3 \mathrm{~b})$. With the needle tip below the interface, $\lambda$ is less sensitive to $h$ (regime III), but increases as the needle tip goes deeper. In the following we focus on rationalizing the case of $h=0$ at the intersection between the regimes II and III.

In Fig. $3 \mathrm{c}$ and $\mathrm{d}$, we plot the wavelength $\lambda$ and the apparent radius $R_{\mathrm{c}}$ versus the needle speed for the various flow rates listed in the legend of Fig. 3d. In all cases, the needle depth is $h=0$ (as marked by a ( ${ }^{*}$ ) in Fig. $3 b$ ). As evident from Fig. 3c we observe that the variation in wavelength $\lambda$ is limited in the range of speeds and flow rates considered in our experiments. The mean value of the wavelength is $\bar{\lambda}=6.1 \mathrm{~mm}$ (plotted as a continuous line in Fig. 3c), while most experiments are within one standard deviation of the mean $\left(\lambda_{ \pm}=2.8\right.$ and $9.4 \mathrm{~mm}$ are plotted as dashed lines in Fig. 3c). Using mass conservation we infer the volume of the droplets $V=Q \lambda / u$. Inserting this quantity in eqn (1), we find that:

$$
R_{\mathrm{c}}=\sqrt{\frac{\Delta \rho}{\pi \rho_{\mathrm{v}}} \frac{\lambda}{\ell}} \sqrt{\frac{Q}{u}}
$$
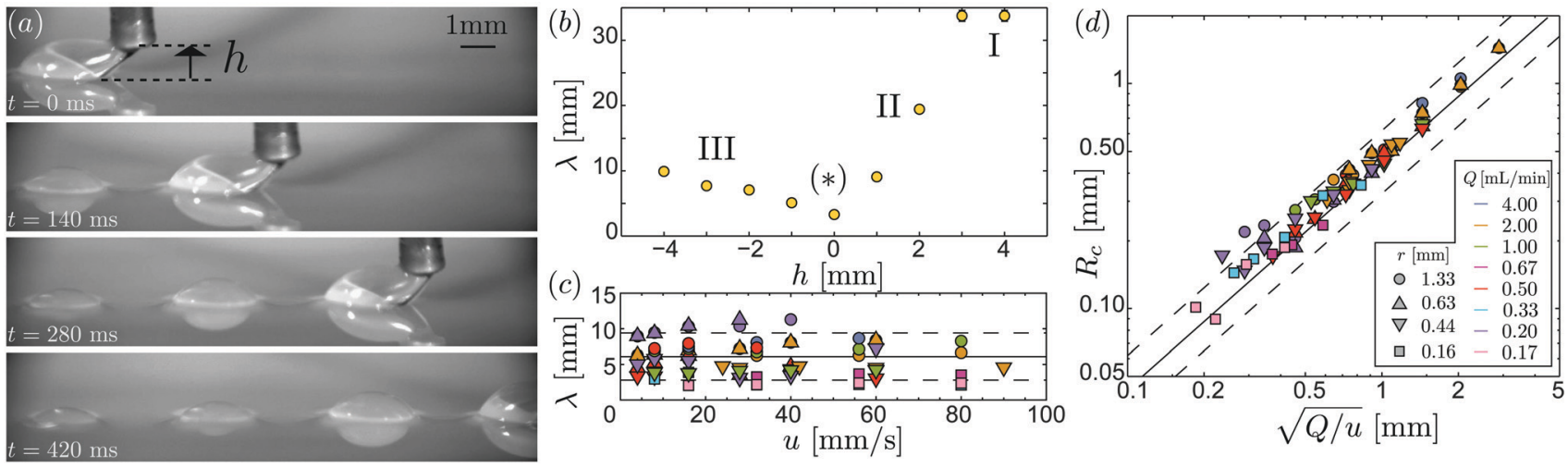

Fig. 3 (a) High speed image sequence $(\Delta t=140 \mathrm{~ms})$ of the droplets deposition. The position of the needle tip relative to the air-elastomer interface is $h=1.0 \mathrm{~mm}$. (b) Distance between droplets $\lambda$ as a function of $h$. (c) Distance between droplets $\lambda$ plotted versus the needle speed $u$. The tip of the needle is positioned at the interface, $h=0$. Volumetric flow rates $Q$ are coded in colors (legend in panel d). (d) Size of the drops characterized by their apparent radius $R_{\mathrm{c}}$ as a function of $(Q / u)^{1 / 2}$. 
In Fig. 3d we show that our experimental data collapse onto a master curve when expressed as a function of $(Q / u)^{1 / 2}$. The scaling prediction in eqn (2) obtained using the wavelengths observed experimentally, is shown as a continuous line $(\bar{\lambda}=6.1 \mathrm{~mm})$ and two dashed lines correspond to the same expression obtained using the values of $\lambda_{ \pm}$. These lines are in good agreement with the experimental data, such that the drop size and the pattern wavelength can be tuned by changing the values of $Q$ and $u$ while keeping the same nozzle.

In our experiments, we observe that before curing is complete, the liquid elastomer slowly climbs upslope onto the water droplet. For small enough droplets $(V \sim 10 \mu \mathrm{L})$, a thin layer of elastomer may cover the whole droplet. This is known as the cloaking process. ${ }^{40-42}$ Yet, in most cases with bigger droplets the cloaking film is incomplete. In order to better encapsulate our droplets, we propose to operate in the regime III (as defined in Fig. 3b) where droplets are produced below the free surface. In such a case, curing freezes the position of the droplets at a given distance $H$ from the interface. After detaching from the nozzle, the water droplets rise towards the air-elastomer interface and the viscous liquid elastomer drains on top of the droplets, thereby setting the final membrane thickness $H$ when curing is complete. ${ }^{43-45}$ In Fig. 4a, we show that $H$ can be tuned through a simple change in the injection depth $h$. These membranes formed with this method fully encapsulate the drops and protect them from outside pollutants, e.g. dust. Yet, the liquid may still be accessed, either via diffusion through the membrane or else by puncturing the membrane as discussed next. (a)
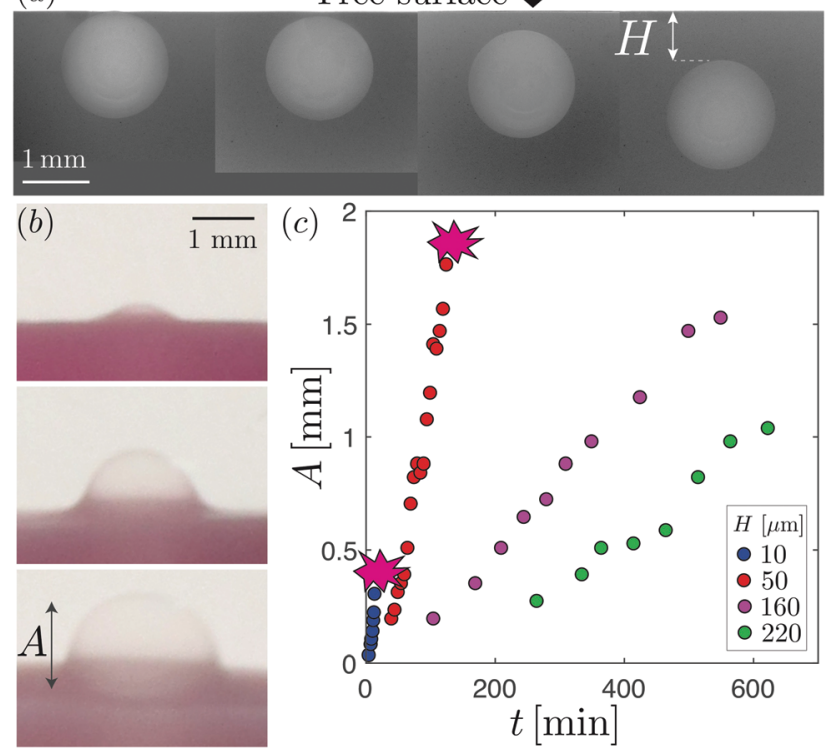

Fig. 4 (a) Cross-section of the encapsulated droplets with the same diameter $d=1.6 \mathrm{~mm}$ but different printing depth $h$ and thus film thickness $H$. (b) Snapshots of a protruding droplet with initial diameter $d=1.6 \mathrm{~mm}$, film thickness $H=160 \mu \mathrm{m}$ at time $t=180,360$ and $540 \mathrm{~min}$ in an ethano bath. (c) Amplitude, $A$, of protruding droplets of initial diameter $d=1.6 \mathrm{~mm}$ in an ethanol bath versus time for different film thicknesses. The pink symbol indicates the rupture of the droplet.
In Fig. $4 \mathrm{~b}$ we show the photographs of a single encapsulated water drop placed in an ethanol bath. After the drop is immersed we observe that the encapsulated drop increases in volume as evident from the bulge. We argue that this change in volume is caused by the diffusion of ethanol through the membrane. ${ }^{46,47}$ In Fig. 4c, we report the change in amplitude, $A$, of the drop as a function of time, for four different capsules. The capsules shown in the plot merely differ in membrane thickness $H$. Thin films $(H<90 \mu \mathrm{m})$ yield a rapid diffusion but eventually burst. Thicker membranes inflate slower but resist to large deformations. Thus, tailoring the film thickness, via a change in print depth, provides control in the dynamics of swelling of our encapsulated drops. We now focus on droplets with film thickness $(90<H[\mu \mathrm{m}]<160)$ that presents the fastest actuation without rupture.

We take advantage of the inflation of encapsulated droplets at the surface of our elastomers to mimic the differential growth of biological tissues. ${ }^{48-50}$ In Fig. 5a, we show the dynamics of a beam with embedded water droplets at its upper surface, which is submerged in ethanol. Over time, the embedded droplets swell much faster than the rest of the beam, inducing an effective growth in the beam upper layer. As a consequence, the beam bends ${ }^{51-54}$ and eventually coils into a loop. Placing the sample into water reverses the motion of the beam, as ethanol diffuses back into the bath causing the
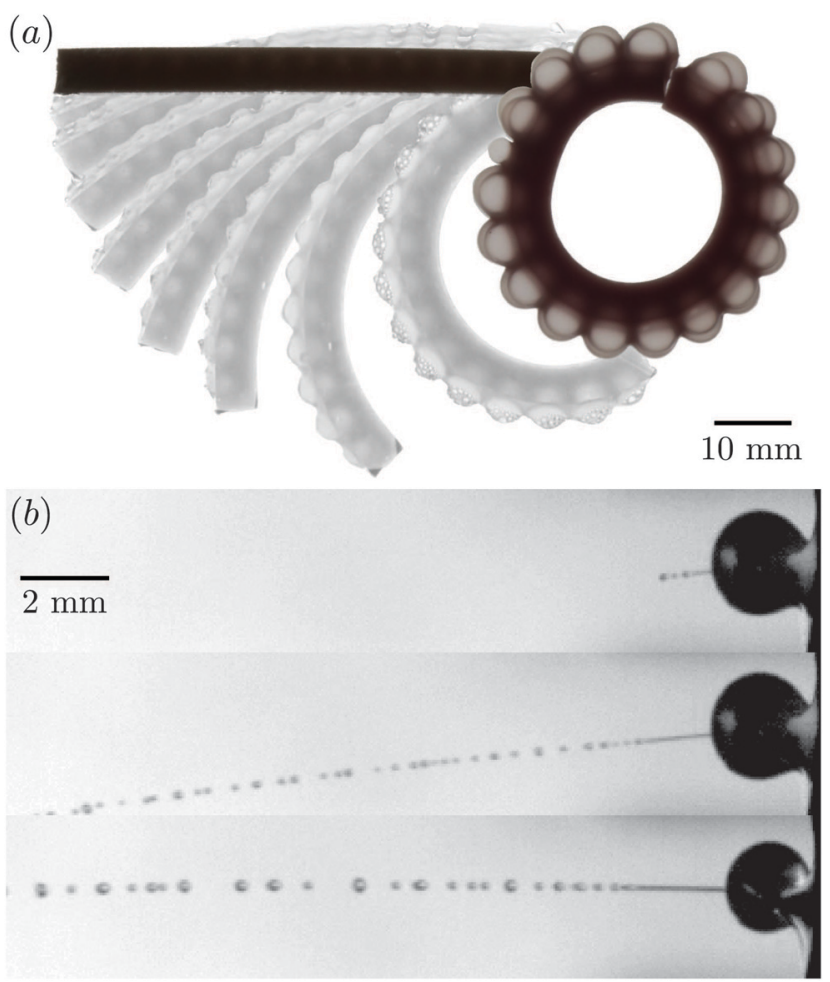

Fig. 5 (a) Bending of a beam. The straight beam with encapsulated water droplets is placed in an ethanol bath for 12 hours. The droplets swell as ethanol diffuses into them, bending the beam into a loop. (b) Bursting of an encapsulated drop under compression. Photographs of the ensuing jet taken at time $t=0.02,0.21$ and $4.13 \mathrm{~s}$ after the onset of the rupture. 
drops to return to their initial state. This experiment shows that it is possible to control the local swelling properties of a solid by printing water drops at its interface using our technique. Each capsule acts as a hinge that can be distributed at the surface of a slender body so as to obtain a desired deformation. Timing successive deformations is also theoretically possible through variations of the thickness of the membrane of the capsule. Thinner membranes would bulge first, while thicker capsules would only bulge at a later time, thereby producing untethered programmable soft robots. ${ }^{55}$ Apart from this type of actuating functionality, our embedded droplets can also be used as small liquid reservoirs encapsulated at the surface of the elastic matrix.

From cells to water balloons, capsules are ubiquitous in our lives, where they enable the transport and delivery of liquids, otherwise shapeless and prone to contamination. In the laboratory, submillimetric capsules are typically fabricated using microfluidic devices operating with low viscosity fluids. ${ }^{56}$ Our freeform approach is complementary as it enables the encapsulation of millimetric drops, which can release their content explosively akin the bursting of oil gland reservoirs in citrus exocarp. ${ }^{57}$ In Fig. 5b, we provide photographs of an encapsulated water droplet compressed with a universal force testing machine (Instron 5944, with a $10 \mathrm{kN}$ load cell). The elastic sample is placed between platens and uniaxial compression is applied steadily up to the point where the membrane of the capsule ruptures. Fig. 5b shows images of the resulting jet observed with a high-speed camera. The microjet (radius $100 \mu \mathrm{m})$ forms through a pore in the membrane and shoots the liquid at high speed (jet speed $\sim 3 \mathrm{~m} \mathrm{~s}^{-1}$ ).

In summary, we have introduced a droplet-based strategy to pattern the shape of an air-elastomer interface. We rationalized the shapes adopted by the water droplets at this interface using the framework of fluid mechanics, thus providing a guide for the selection of printing parameters to achieve the desired distribution of surface cavities. We have also shown that the encapsulation of water droplets can be obtained and enables the functionalization of the elastomer. The drops can be used to actuate the beam via swelling, such that printing a drop pattern can program the deformation of slender elastomeric structures. These encapsulated drops can also be used as micro-reservoirs, transporting and protecting minute quantities of liquid, which can be released when puncturing the membrane of the capsule. We envision that our work will be impactful as it provides a simple and robust methodology to fabricate architected and thus functional elastomers.
1 P. Vukusic and J. Sambles, Nature, 2003, 424, 852.

2 M. Land, Prog. Biophys. Mol. Biol., 1972, 24, 75-106.

3 J. Hazel, M. Stone, M. Grace and V. Tsukruk, J. Biomech., 1999, 32, 477-484.

4 M. Clauss, R. Hofmann, W. Streich, J. Fickel and J. Hummel, J. Zool., 2010, 281, 26-38.

5 M. Kolle, P. Salgard-Cunha, M. Scherer, F. Huang, P. Vukusic, S. Mahajan, J. Baumberg and U. Steiner, Nat. Nanotechnol., 2010, 5, 511-515.

6 M. Honda, T. Seki and Y. Takeoka, Adv. Mater., 2009, 21, 1801-1804.

7 K. Sumioka, H. Kayashima and T. Tsutsui, Adv. Mater., 2002, 14, 1284-1286.

8 H. Fudouzi and T. Sawada, Langmuir, 2006, 22, 1365-1368.

9 T. Brunet, J. Leng and O. Mondain-Monval, Science, 2013, 342, 323-324.

10 Q. Zhang, X. Yang, P. Li, G. Huang, S. Feng, C. Shen, B. Han, X. Zhang, F. Jin and F. Xu, et al., Prog. Mater. Sci., 2015, 74, 332-400.

11 S. Lee, H. Kim, W. Choi, G. Yoon and E. Seo, Soft Matter, 2019, 15, 8459-8467.

12 K. Bertoldi, V. Vitelli, J. Christensen and M. van Hecke, Nat. Rev. Mater., 2017, 2, 17066.

13 G. Koenderink, Z. Dogic, F. Nakamura, P. Bendix, F. MacKintosh, J. Hartwig, T. Stossel and D. Weitz, Proc. Natl. Acad. Sci. U. S. A., 2009, 106, 15192-15197.

14 C. Schumacher, B. Bickel, J. Rys, S. Marschner, C. Daraio and M. Gross, ACM Trans. Graph., 2015, 34, 136.

15 B. Florijn, C. Coulais and M. van Hecke, Phys. Rev. Lett., 2014, 113, 175503.

16 J. Li, T. Pallicity, V. Slesarenko, A. Goshkoderia and S. Rudykh, Adv. Mater., 2019, 31, 1807309.

17 R. Truby and J. Lewis, Nature, 2016, 540, 371-378.

18 B.-J. De Gans, P. Duineveld and U. Schubert, Adv. Mater., 2004, 16, 203-213.

19 K. Hajash, B. Sparrman, C. Guberan, J. Laucks and S. Tibbits, 3D Print. Addit. Manuf., 2017, 4, 123-132.

20 J. Kaufman, G. Tao, S. Shabahang, E.-H. Banaei, D. Deng, X. Liang, S. Johnson, Y. Fink and A. Abouraddy, Nature, 2012, 487, 463-467.

21 J. Kaufman, R. Ottman, G. Tao, S. Shabahang, E.-H. Banaei, X. Liang, S. Johnson, Y. Fink, R. Chakrabarti and A. Abouraddy, Proc. Natl. Acad. Sci. U. S. A., 2013, 110, 15549-15554.

22 L. Cai, J. Marthelot and P.-T. Brun, Proc. Natl. Acad. Sci. U. S. A., 2019, 116, 22966-22971.

23 D. Kolesky, R. Truby, A. Gladman, T. Busbee, K. Homan and J. Lewis, Adv. Mater., 2014, 26, 3124-3130.

24 S. Murphy and A. Atala, Nat. Biotechnol., 2014, 32, 773.

25 Y. Rim, S.-H. Bae, H. Chen, N. De Marco and Y. Yang, Adv. Mater., 2016, 28, 4415-4440.

26 R. Style, R. Boltyanskiy, B. Allen, K. Jensen, H. Foote, J. Wettlaufer and E. Dufresne, Nat. Phys., 2015, 11, 82.

27 P. Testa, R. Style, J. Cui, C. Donnelly, E. Borisova, P. Derlet, E. Dufresne and L. Heyderman, Adv. Mater., 2019, 1900561. 
28 M. Bartlett, A. Fassler, N. Kazem, E. Markvicka, P. Mandal and C. Majidi, Adv. Mater., 2016, 28, 3726-3731.

29 M. Bartlett, N. Kazem, M. Powell-Palm, X. Huang, W. Sun, J. Malen and C. Majidi, Proc. Natl. Acad. Sci. U. S. A., 2017, 114, 2143-2148.

30 A. Miriyev, L. Stack and H. Lipson, Nat. Commun., 2017, 8, 596.

31 Z. Yang, J. Wei, Y. Sobolev and B. Grzybowski, Nature, 2018, $553,313$.

32 G. Whitesides, Nature, 2006, 442, 368.

33 T. Sanchez, D. Chen, S. DeCamp, M. Heymann and Z. Dogic, Nature, 2012, 491, 431.

34 S. Shi, X. Liu, Y. Li, X. Wu, D. Wang, J. Forth and T. Russell, Adv. Mater., 2018, 30, 1705800.

35 J. Forth, X. Liu, J. Hasnain, A. Toor, K. Miszta, S. Shi, P. Geissler, T. Emrick, B. Helms and T. Russell, Adv. Mater., 2018, 30, 1707603.

36 P.-G. De Gennes, F. Brochard-Wyart and D. Quéré, Capillarity and wetting phenomena: drops, bubbles, pearls, waves, Springer Science \& Business Media, 2013.

37 O. Del Ro and A. Neumann, J. Colloid Interface Sci., 1997, 196, 136-147.

38 W. Harkins and E. Humphery, J. Am. Chem. Soc., 1916, 38, 228-236.

39 M. Wilkinson, J. Colloid Interface Sci., 1972, 40, 14-26.

40 F. Schellenberger, J. Xie, N. Encinas, A. Hardy, M. Klapper, P. Papadopoulos, H.-J. Butt and D. Vollmer, Soft Matter, 2015, 11, 7617-7626.

41 S. Anand, K. Rykaczewski, S. Subramanyam, D. Beysens and K. Varanasi, Soft Matter, 2015, 11, 69-80.
42 I. Guha, S. Anand and K. Varanasi, Nat. Commun., 2017, 8, 1371.

43 A. Lee, P.-T. Brun, J. Marthelot, G. Balestra, F. Gallaire and P. Reis, Nat. Commun., 2016, 7, 11155.

44 J. Marthelot, E. Strong, P. M. Reis and P.-T. Brun, Nat. Commun., 2018, 9, 4477.

45 J. Schleifer, J. Marthelot, T. Jones and P.-T. Brun, Soft Matter, 2019, 15, 1405-1412.

46 Y. Lee, D. Bourgeois and G. Belfort, J. Membr. Sci., 1989, 44, 161-181.

47 P. Peng, B. Shi and Y. Lan, Sep. Sci. Technol., 2010, 46, 234-246.

48 Y. Klein, E. Efrati and E. Sharon, Science, 2007, 315, 1116-1120.

49 L. Huang, R. Jiang, J. Wu, J. Song, H. Bai, B. Li, Q. Zhao and T. Xie, Adv. Mater., 2017, 29, 1605390.

50 E. Siéfert, E. Reyssat, J. Bico and B. Roman, Nat. Mater., 2019, 18, 24.

51 H. Yuk and X. Zhao, Adv. Mater., 2018, 30, 1704028.

52 A. Gladman, E. Matsumoto, R. Nuzzo, L. Mahadevan and J. Lewis, Nat. Mater., 2016, 15, 413.

53 A. Lee, D. Yan, M. Pezzulla, D. Holmes and P. Reis, Soft Matter, 2019, 15, 6134-6144.

54 M. Pezzulla, G. Smith, P. Nardinocchi and D. Holmes, Soft Matter, 2016, 12, 4435-4442.

55 S. Rich, R. Wood and C. Majidi, Nat. Electron., 2018, 1, 102-112.

56 A. Utada, E. Lorenceau, D. Link, P. Kaplan, H. A. Stone and D. Weitz, Science, 2005, 308, 537-541.

57 N. Smith, H. Ebrahimi, R. Ghosh and A. Dickerson, Proc. Natl. Acad. Sci. U. S. A., 2018, 115, E5887-E5895. 